\title{
Higher dimensional bianchi type-V universe in creation-field cosmology
}

\author{
Kishor S. Adhav ${ }^{*}$, Shivdas D. Katore, Abhijit S. Bansod, Prachi S. Gadodia
}

Department of Mathematics, Sant Gadge Baba Amravati University, Amravati, India;

*Corresponding Author: ati ksadhav@yahoo.co.in

Received 16 December 2009; revised 6 March 2010; accepted 15 March 2010.

\begin{abstract}
We have studied the Hoyle-Narlikar $C$-field cosmology with Bianchi type-V non static spacetime in higher dimensions. Using methods of Narlikar and Padmanabham [1], the solutions have been studied when the creation field $C$ is a function of time $t$ only as space time is non static. The geometrical and physical aspects for model are also studied.
\end{abstract}

Keywords: Bianchi Type-V Space-Time, Creation Field Cosmology, Cosmological Model of Universe, Higher Dimensions

\section{INTRODUCTION}

The study of higher dimensional physics is important because of several prominent results obtained in the development of the super-string theory. In the latest study of super-strings and super-gravity theories, Weinberg [2] studied the unification of the fundamental forces with gravity, which reveals that the space-time should be different from four. Since the concept of higher dimensions is not unphysical, the string theories are discussed in 10dimensions and 26-dimensions of space-time. Because of this, many researchers are inspired to study the higher dimensional to explore the hidden knowledge of the universe. Chodos and Detweller [3], Lorentz-Petzold [4], Ibanez and Verdaguer [5], Gleiser and Diaz [6], Banerjee and Bhui [7], Reddy and Venkateswara [8], Khadekar and Gaikwad [9], Adhav et al. [10] have studied the multi-dimensional cosmological models in general relativity and in other alternative theories of gravitation.

The three important observations in astronomy namely the phenomenon of expanding universe, primordial nucleon-synthesis and the observed isotropy of cosmic microwave background radiation (CMBR) were supposed to be successfully explained by big-bang cosmology based on Einstein's field equations. However, Smoot et al. [11] revealed that the earlier predictions of the Friedman-Robertson-Walker type of models do not always exactly meet our expectations. Some puzzling results regarding the red shifts from the extra galactic objects continue to contradict the theoretical explanations given from the big bang type of the model. Also, CMBR discovery did not prove it to be a out come of big bang theory. Infact, Narlikar et al. [12] have proved the possibility of non-relic interpretation of CMBR. To explain such phenomenon, many alternative theories have been proposed from time to time. Hoyle [13], Bondi and Gold [14] proposed steady state theory in which the universe does not have singular beginning nor an end on the cosmic time scale. Moreover, they have shown that the statistical properties of the large scale features of the universe do not change. Further, the constancy of the mass density has been accounted by continuous creation of matter going on in contrast to the one time infinite and explosive creation of matter at $t=0$ as in the earlier standard model. But the principle of conservation of matter was violated in this formalism. To overcome this difficulty Hoyle and Narlikar [15] adopted a field theoretic approach by introducing a mass less and charge less scalar field $C$ in the Einstein-Hilbert action to account for the matter creation. In the $C$-field theory introduced by Hoyle and Narlikar there is no big bag type of singularity as in the steady state theory of Bondi and Gold [14]. A solution of Einstein's field equations admitting radiation with negative energy mass less scalar creation fields $C$ was obtained by Narlikar and Padmanabhan [1]. The study of Hoyle and Narlikar theory [15-17] to the space-time of dimensions more than four was carried out by Chatterjee and Banerjee [18]. The solutions of Einstein's field equations in the presence of creation field have been obtained for Bianchi type- $\mathrm{V}$ universe in four dimensions by Singh and Chaubey [19].

Here, we have considered a spatially homogeneous and anisotropic non static Bianchi type- $\mathrm{V}$ cosmological model in Hoyle and Narlikar $C$-field cosmology with five dimensions. Therefore, we have assumed that the creation field $C$ is a function of time $t$ only i.e. 
$C(x, t)=C(t)$.

This study is important because of the fact that the resulting cosmological model is considered to be amenable to the model obtained by Singh and Chaubey [19].

\section{HOYLE AND NARLIKAR C-FIELD COSMOLOGY}

Introducing a mass less scalar field called as creation field namely $C$-field, Einstein's field equations are modified. Hoyle and Narlikar [15-17] field equations are

$$
R_{i j}-\frac{1}{2} g_{i j} R=-8 \pi\left({ }^{m} T_{i j}+{ }^{c} T_{i j}\right)
$$

where ${ }^{m} T_{i j}$ is matter tensor of Einstein theory and ${ }^{c} T_{i j}$ is matter tensor due to the $C$-field which is given by

$$
{ }^{c} T_{i j}=-f\left(C_{i} C_{j}-\frac{1}{2} g_{i j} C^{k} C_{k}\right)
$$

where $f>0$ and $C_{i}=\frac{\partial C}{\partial x^{i}}$.

Because of the negative value of $T^{00}\left(T^{00}<0\right)$, the $C$-field has negative energy density producing repulsive gravitational field which causes the expansion of the universe. Hence, the energy conservation equation reduces to

$$
{ }^{m} T_{; j}^{i j}=-{ }^{c} T_{; j}^{i j}=f C^{i} C^{j} ; j
$$

i.e. matter creation through non-zero left hand side is possible while conserving the over all energy and momentum.

Above equation is similar to

$$
m g_{i j} \frac{d x^{i}}{d s}-C_{j}=0
$$

which implies that the 4-momentum of the created particle is compensated by the 4-momentum of the $C$-field. In order to maintain the balance, the $C$-field must have negative energy. Further, the $C$-field satisfy the source equation $f C_{; i}^{i}=J_{; i}^{i}$ and $J^{i}=\rho \frac{d x^{i}}{d s}=\rho v^{i}$, where $\rho$ is homogeneous mass density.

\section{METRIC AND FIELD EQUATIONS}

The five-dimensional Bianchi-Type- $\mathrm{V}$ line element can be written as

$d s^{2}=d t^{2}-a_{1}{ }^{2} d x^{2}-a_{2}{ }^{2} e^{-2 m x} d y^{2}-a_{3}{ }^{2} e^{-2 m x} d z^{2}-a_{4}{ }^{2} e^{-2 m x} d u^{2}$

where $a_{1}, a_{2}, a_{3}$ and $a_{4}$ are functions of $t$ only and $m$ is constant.

Here the extra coordinate is taken to be space like.
The above space time is non static, hence, we have assumed that creation field $C$ is function of time $t$ only i.e.

$$
C(x, t)=C(t) \text { and }{ }^{m} T_{j}^{i}=\operatorname{diag}(\rho,-p,-p,-p,-p)
$$

We have assumed that velocity of light and gravitational constant are equal to one unit.

Now, the Hoyle-Narlikar field Eq.1 for metric (5) with the help of Eqs.2, 3, and $\mathbf{6}$ can be written as

$$
\begin{aligned}
& \frac{\dot{a}_{1} \dot{a}_{2}}{a_{1} a_{2}}+\frac{\dot{a}_{1} \dot{a}_{3}}{a_{1} a_{3}}+\frac{\dot{a}_{1} \dot{a}_{4}}{a_{1} a_{4}}+\frac{\dot{a}_{2} \dot{a}_{3}}{a_{2} a_{3}}+\frac{\dot{a}_{2} \dot{a}_{4}}{a_{2} a_{4}}+\frac{\dot{a}_{3} \dot{a}_{4}}{a_{3} a_{4}}-\frac{6 m^{2}}{a_{1}^{2}} \\
= & 8 \pi\left(\rho-\frac{1}{2} f \dot{C}^{2}\right) \\
& \frac{\ddot{a}_{2}}{a_{2}}+\frac{\ddot{a}_{3}}{a_{3}}+\frac{\ddot{a}_{4}}{a_{4}}+\frac{\dot{a}_{2} \dot{a}_{3}}{a_{2} a_{3}}+\frac{\dot{a}_{2} \dot{a}_{4}}{a_{2} a_{4}}+\frac{\dot{a}_{3} \dot{a}_{4}}{a_{3} a_{4}}-\frac{3 m^{2}}{a_{1}^{2}} \\
= & 8 \pi\left(-p+\frac{1}{2} f \dot{C}^{2}\right) \\
& \frac{\ddot{a}_{1}}{a_{1}}+\frac{\ddot{a}_{3}}{a_{3}}+\frac{\ddot{a}_{4}}{a_{4}}+\frac{\dot{a}_{1} \dot{a}_{3}}{a_{1} a_{3}}+\frac{\dot{a}_{1} \dot{a}_{4}}{a_{1} a_{4}}+\frac{\dot{a}_{3} \dot{a}_{4}}{a_{3} a_{4}}-\frac{3 m^{2}}{a_{1}^{2}} \\
= & 8 \pi\left(-p+\frac{1}{2} f \dot{C}^{2}\right) \\
& \frac{\ddot{a}_{1}}{a_{1}}+\frac{\ddot{a}_{2}}{a_{2}}+\frac{\ddot{a}_{4}}{a_{4}}+\frac{\dot{a}_{1} \dot{a}_{2}}{a_{1} a_{2}}+\frac{\dot{a}_{1} \dot{a}_{4}}{a_{1} a_{4}}+\frac{\dot{a}_{2} \dot{a}_{4}}{a_{2} a_{4}}-\frac{3 m^{2}}{a_{1}^{2}} \\
= & 8 \pi\left(-p+\frac{1}{2} f \dot{C}^{2}\right) \\
& =f \dot{C}\left(\frac{\ddot{C}^{2}}{a_{1}}+\left(\frac{\dot{a}_{1}}{a_{1}}+\frac{\dot{a}_{2}}{a_{2}}+\frac{\dot{a}_{3}}{a_{3}}+\frac{\dot{a}_{4}}{a_{4}}\right) \dot{C} \dot{a}_{2} \ddot{a}_{3}\right. \\
& \frac{\ddot{a}_{1}}{a_{2}}+\frac{\dot{a}_{1} \dot{a}_{2}}{a_{1} a_{2}}+\frac{\dot{a}_{1} \dot{a}_{3}}{a_{1} a_{3}}+\frac{\dot{a}_{2} \dot{a}_{3}}{a_{2} a_{3}}-\frac{3 m^{2}}{a_{1}^{2}} \\
= & 8 \pi\left(-p+\frac{\dot{a}_{3}}{a_{3}}+\frac{\dot{a}_{4}}{a_{4}}\right)\left(\dot{C}^{2}\right) \\
& \left.3 \frac{\dot{a}_{1}}{a_{1}}=\frac{\dot{a}_{2}}{a_{2}}+\frac{\dot{a}_{3}}{a_{3}}+\frac{\dot{a}_{4}}{a_{4}}+p\right) \\
&
\end{aligned}
$$

where dot $(\cdot)$ indicates the derivative with respect to $t$. From Eq.12, we get

$$
a_{1}^{3}=a_{2} a_{3} a_{4}
$$

Assume that $V$ is a function of time $t$ defined by

$$
V=a_{1} a_{2} a_{3} a_{4}
$$

From Eqs.14 and 15, we get 


$$
a_{1}=V^{\frac{1}{4}}
$$

Above Eq.13 can be written in the form

$$
\frac{d}{d V}(V \rho)+p=f \dot{C}(V) \frac{d}{d V}[V \dot{C}(V)]
$$

In order to obtain a unique solution, one has to specify the rate of creation of matter-energy (at the expense of the negative energy of the $C$-field). Without loss of generality, we assume that the rate of creation of matter energy density is proportional to the strength of the existing $C$-field energy-density. i.e. the rate of creation of matter energy density per unit proper-volume is given by

$$
\frac{d}{d V}(V \rho)+p=\alpha^{2} \dot{C}^{2} \equiv \alpha^{2} g^{2}(V)
$$

where $\alpha$ is proportionality constant and we have defined $\dot{C}(V) \equiv g(V)$.

Substituting it in Eq.17, we get

$$
\frac{d}{d V}(V \rho)+p=f g(V) \frac{d}{d V}(V g)
$$

Comparing right hand sides of Eqs.18 and 19, we get

$$
g(V) \frac{d}{d V}(g V)=\frac{\alpha^{2}}{f} g^{2}(V)
$$

Integrating, which gives

$$
g(V)=A_{1} V^{\left(\frac{\alpha^{2}}{f}-1\right)}
$$

where $A_{1}$ is arbitrary constant of integration.

We consider the equation of state of matter as

$$
p=\gamma \rho
$$

Substituting Eqs.21 and 22 in the Eq.18, we get

$$
\frac{d}{d V}(V \rho)+\gamma \rho=\alpha^{2} A_{1}^{2} V^{2\left(\frac{\alpha^{2}}{f}-1\right)}
$$

which further yields

$$
\rho=\frac{\alpha^{2} A_{1}^{2}}{\left(2 \frac{\alpha^{2}}{f}-1+\gamma\right)} V^{2\left(\frac{\alpha^{2}}{f}-1\right)}
$$

From Eq.22, we get

$$
p=\frac{\alpha^{2} A_{1}^{2} \gamma}{\left(2 \frac{\alpha^{2}}{f}-1+\gamma\right)} V^{2\left(\frac{\alpha^{2}}{f}-1\right)}
$$

\footnotetext{
Subtracting Eq.8 from Eq.9, we get
}

$$
\frac{d}{d t}\left(\frac{\dot{a}_{1}}{a_{1}}-\frac{\dot{a}_{2}}{a_{2}}\right)+\left(\frac{\dot{a}_{1}}{a_{1}}-\frac{\dot{a}_{2}}{a_{2}}\right)\left(\frac{\dot{a}_{1}}{a_{1}}+\frac{\dot{a}_{2}}{a_{2}}+\frac{\dot{a}_{3}}{a_{3}}+\frac{\dot{a}_{4}}{a_{4}}\right)=0
$$

Now, from Eqs.15 and 26, we get

$$
\frac{d}{d t}\left(\frac{\dot{a}_{1}}{a_{1}}-\frac{\dot{a}_{2}}{a_{2}}\right)+\left(\frac{\dot{a}_{1}}{a_{1}}-\frac{\dot{a}_{2}}{a_{2}}\right) \frac{\dot{V}}{V}=0
$$

Integrating, which gives

$$
\frac{a_{1}}{a_{2}}=d_{1} \exp \left(x_{1} \int \frac{d t}{V}\right), d_{1}=\text { constant }, x_{1}=\text { constant }
$$

Subtracting Eq.9 from Eq.10, we get

$$
\frac{d}{d t}\left(\frac{\dot{a}_{2}}{a_{2}}-\frac{\dot{a}_{3}}{a_{3}}\right)+\left(\frac{\dot{a}_{2}}{a_{2}}-\frac{\dot{a}_{3}}{a_{3}}\right) \frac{\dot{V}}{V}=0
$$

Integrating, we get

$$
\frac{a_{2}}{a_{3}}=d_{2} \exp \left(x_{2} \int \frac{d t}{V}\right), d_{2}=\text { constant }, x_{2}=\text { constant }
$$

Subtracting Eq.10 from Eq.11, we get

$$
\frac{d}{d t}\left(\frac{\dot{a}_{3}}{a_{3}}-\frac{\dot{a}_{4}}{a_{4}}\right)+\left(\frac{\dot{a}_{3}}{a_{3}}-\frac{\dot{a}_{4}}{a_{4}}\right) \frac{\dot{V}}{V}=0
$$

which on integration gives

$$
\frac{a_{3}}{a_{4}}=d_{3} \exp \left(x_{3} \int \frac{d t}{V}\right), d_{3}=\text { constant }, x_{3}=\text { constant }
$$

Subtracting Eq.8 from Eq.11, we get

$$
\frac{d}{d t}\left(\frac{\dot{a}_{1}}{a_{1}}-\frac{\dot{a}_{4}}{a_{4}}\right)+\left(\frac{\dot{a}_{1}}{a_{1}}-\frac{\dot{a}_{4}}{a_{4}}\right) \frac{\dot{V}}{V}=0
$$

Integrating, we get

$$
\frac{a_{1}}{a_{4}}=d_{4} \exp \left(x_{4} \int \frac{d t}{V}\right), d_{4}=\text { constant, } x_{4}=\text { constant }
$$

where $d_{4}=d_{1} d_{2} d_{3}, x_{4}=x_{1}+x_{2}+x_{3}$ and $V=a_{1} a_{2} a_{3} a_{4}$.

Using Eqs.27, 28, 29 and 30, the values of $a_{1}(t)$, $a_{2}(t), a_{3}(t)$ and $a_{4}(t)$ can be written explicitly as

$$
\begin{aligned}
& a_{1}(t)=D_{1} V^{1 / 4} \exp \left(X_{1} \int \frac{d t}{V}\right) \\
& a_{2}(t)=D_{2} V^{1 / 4} \exp \left(X_{2} \int \frac{d t}{V}\right) \\
& a_{3}(t)=D_{3} V^{1 / 4} \exp \left(X_{3} \int \frac{d t}{V}\right)
\end{aligned}
$$




$$
a_{4}(t)=D_{4} V^{1 / 4} \exp \left(X_{4} \int \frac{d t}{V}\right)
$$

where the relations $D_{1} D_{2} D_{3} D_{4}=1$ and $X_{1}+X_{2}+$ $X_{3}+X_{4}=0$ are satisfied by $D_{i}(i=1,2,3,4)$ and $X_{i}(i=1,2,3,4)$. From Eqs.10 and 31, we get $D_{1}=1$ and $X_{1}=0$.

Adding Eqs.8, 9, 10, 11 and 4 times Eq.7, we get

$$
\begin{gathered}
\left(\frac{\ddot{a}_{1}}{a_{1}}+\frac{\ddot{a}_{2}}{a_{2}}+\frac{\ddot{a}_{3}}{a_{3}}+\frac{\ddot{a}_{4}}{a_{4}}\right) \\
+2\left(\frac{\dot{a}_{1} \dot{a}_{2}}{a_{1} a_{2}}+\frac{\dot{a}_{2} \dot{a}_{3}}{a_{2} a_{3}}+\frac{\dot{a}_{3} \dot{a}_{1}}{a_{3} a_{1}}+\frac{\dot{a}_{1} \dot{a}_{4}}{a_{1} a_{4}}+\frac{\dot{a}_{2} \dot{a}_{4}}{a_{2} a_{4}}+\frac{\dot{a}_{3} \dot{a}_{4}}{a_{3} a_{4}}\right) \\
-\frac{12 m^{2}}{a_{1}^{2}}=\frac{32}{3} \pi(\rho-p)
\end{gathered}
$$

From Eq.15, we have

$$
\begin{aligned}
& \frac{\ddot{V}}{V}=\left(\frac{\ddot{a}_{1}}{a_{1}}+\frac{\ddot{a}_{2}}{a_{2}}+\frac{\ddot{a}_{3}}{a_{3}}+\frac{\ddot{a}_{4}}{a_{4}}\right) \\
& +2\left(\frac{\dot{a}_{1} \dot{a}_{2}}{a_{1} a_{2}}+\frac{\dot{a}_{2} \dot{a}_{3}}{a_{2} a_{3}}+\frac{\dot{a}_{3} \dot{a}_{1}}{a_{3} a_{1}}+\frac{\dot{a}_{1} \dot{a}_{4}}{a_{1} a_{4}}+\frac{\dot{a}_{2} \dot{a}_{4}}{a_{2} a_{4}}+\frac{\dot{a}_{3} \dot{a}_{4}}{a_{3} a_{4}}\right)
\end{aligned}
$$

From Eqs.35, 36 and 22, we get

$$
\frac{\ddot{V}}{V}-\frac{12 m^{2}}{a_{1}^{2}}=\frac{32}{3} \pi(1-\gamma) \rho
$$

Substituting Eq.16 in Eq.37, we get

$$
\frac{\ddot{V}}{V}-\frac{12 m^{2}}{V^{1 / 2}}=\frac{32}{3} \pi(1-\gamma) \rho
$$

Substituting Eq.24 in Eq.38, we get

$$
\frac{\ddot{V}}{V}-\frac{12 m^{2}}{V^{1 / 2}}=\frac{32}{3} \pi(1-\gamma) \frac{\alpha^{2} A^{2}}{\left(2 \frac{\alpha^{2}}{f}-1+\gamma\right)} V^{2\left(\frac{\alpha^{2}}{f}-1\right)}
$$

which further gives

$$
\int \frac{d V}{\sqrt{\frac{32 \pi(1-\gamma) A^{2} f}{3\left(2 \frac{\alpha^{2}}{f}-1+\gamma\right)} V^{2 \alpha^{2} / f}+16 m^{2} V^{3 / 2}+k_{1}}}=t
$$

where $k_{1}$ is integration constant.

For $\gamma=1$ (Zeldovich fluid or Stiff fluid) and $k_{1}=0$, the above equation gives

$$
V=m^{4} t^{4}
$$

Substituting Eq.28 in Eq.21, we get

$$
g=A_{1} m^{4\left(\frac{\alpha^{2}}{f}-1\right)} t^{4\left(\frac{\alpha^{2}}{f}-1\right)}
$$

Also, from equation $\dot{C}(V)=g(V)$, we get

$$
C=\frac{A_{1} m^{4\left(\frac{\alpha^{2}}{f}-1\right)} t^{\left(4 \frac{\alpha^{2}}{f}-3\right)}}{\left(4 \frac{\alpha^{2}}{f}-3\right)}
$$

Substituting Eq.41 in Eq.24, the homogeneous mass density becomes

$$
\rho=\frac{1}{2} A_{1}^{2} f m^{8\left(\frac{\alpha^{2}}{f}-1\right)} t^{8\left(\frac{\alpha^{2}}{f}-1\right)}
$$

Using Eq.25 and $\gamma=1$, pressure becomes

$$
p=\frac{1}{2} A_{1}^{2} f m^{8\left(\frac{\alpha^{2}}{f}-1\right)} t^{8\left(\frac{\alpha^{2}}{f}-1\right)}
$$

From Eqs.38 and 39, it is observed that for $f=\alpha^{2}$, there is no singularity in density and pressure.

Using Eq.41 in Eqs.31, 32, 33 and 34, we get

$$
a_{1}(t)=m t
$$

$$
\begin{aligned}
& a_{2}(t)=D_{2} m t \exp \left(-\frac{X_{2}}{3 m^{4}} \frac{1}{t^{3}}\right) \\
& a_{3}(t)=D_{3} m t \exp \left(-\frac{X_{3}}{3 m^{4}} \frac{1}{t^{3}}\right) \\
& a_{4}(t)=D_{4} m t \exp \left(-\frac{X_{4}}{3 m^{4}} \frac{1}{t^{3}}\right)
\end{aligned}
$$

\section{PHYSICAL PROPERTIES}

The expansion scalar $\theta$ is given by

$$
\theta=4 H=\frac{4}{t}
$$

The mean anisotropy parameter is given by

$$
A=\frac{1}{4} \sum_{i=1}^{4}\left(\frac{\Delta H_{i}}{H}\right)=\frac{X_{2}^{2}+X_{3}^{2}+X_{4}^{2}}{4 m^{8} t^{6}}
$$

The shear scalar $\sigma^{2}$ is given by

$$
\sigma^{2}=\frac{1}{2}\left(\sum_{i=1}^{4} H_{i}^{2}-4 H^{2}\right)=\frac{4}{2} A H^{2}=\frac{X_{2}^{2}+X_{3}^{2}+X_{4}^{2}}{2 m^{8} t^{8}}
$$

The deceleration parameter $q$ is given by

$$
q=\frac{d}{d t}\left(\frac{1}{H}\right)-1=0
$$

where $\Delta H_{i}=H_{i}-H$ and $H$ is the Hubble parameter. 
For large $t$, the expansion scalar and shear scalar tend to zero. Further, if $f>\alpha^{2}$, for large $t$, the model reduces to the vacuum case.

\section{CONCLUSIONS}

In this paper, we have considered the space-time geometry corresponding to Bianchi type- $\mathrm{V}$ in Hoyle-Narlikar [15-17] creation field theory of gravitation with five dimensions. Bianchi type- $\mathrm{V}$ universe in creation-field cosmology has been investigated by Singh and Chaubey [19] whose work has been extended and studied in five dimensions. An attempt has been made to retain Singh and Chaubey's [19] forms of the various quantities. We have noted that all the results of Singh and Chaubey [19] can be obtained from our results by assigning appropriate values to the functions concerned.

\section{REFERENCES}

[1] Narlikar, J.V. and Padmanabhan, T. (1985) Creation-field cosmology: A possible solution to singularity, horizon, and flatness problems. Physical Review D, 32(8), 19281934.

[2] Weinberg, S. (1986) Physics in higher dimensions. World Scientific, Singapore.

[3] Chodos, A. and Detweller, S. (1980) Where has the fifth dimension gone? Physical Review D, 21(8), 2167-2170.

[4] Lorentz-Petzold, K. (1985) Higher-dimensional Brans-Dicke cosmologies. General Relativity and Graviation, 17(7), 1189-1195.

[5] Ibanez, J. and Verdaguer, E. (1986) Radiative isotropic cosmologies with extra dimensions. Physical Review D, 34(12), 1202-1208.

[6] Gleiser, R.J. and Diaz, M.C. (1988) Perfect-fluid cosmologies with extra dimensions. Physical Review D, 37(12), 3761-3764.

[7] Banerjee, S. and Bhui, B. (1990) Homogeneous Cosmological Model in Higher Dimension. Monthly Notices of the Royal Astronomical Society, 247(1), 57-61.

[8] Reddy, D.R.K. and Venkateswara Rao, N. (2001) Some cosmological models in scalar-tensor theory of gravitation. Astrophysics and Space Science, 277(3), 461-472.

[9] Khadekar, G. and Gaikwad, M. (2001) Higher dimensional Bianchi type- $\mathrm{V}$ cosmological model in Bimetric theory of relativity. Proceedings of Einstein Foundation International, 11, 95-100.

[10] Adhav, K., Nimkar, A. and Dawande, M. (2007) N-dimensional string cosmological model in Brans-Dicke theory of gravitation. Astrophysics and Space Science, 310(3-4), 231-235.

[11] Smoot, G.F., et al. (1992) Structure in the COBE differential microwave radiometer first-year maps. Astrophysical Journal, 396(1), 21-25.

[12] Narlikar, J.V., et al. (2003) Inhomogeneities in the microwave background radiation interpreted within the framework of the quasi-steady state cosmology. Astrophysical Journal, 585, 1-11.

[13] Hoyle, F. (1948) A new model for the expanding universe. Monthly Notices of the Royal Astronomical Society, 108(1748), 372-382.

[14] Bondi, H. and Gold, T. (1948) The steady-state theory of the expanding universe. Monthly Notices of the Royal Astronomical Society, 108(3), 252-270.

[15] Hoyle, F. and Narlikar, J.V. (1966) A radical departure from the 'steady-state' concept in cosmology. Proeedings of Royal Society (London) A, 290(1421), 162-176.

[16] Hoyle, F. and Narlikar, J.V. (1963) Mach's principle and the creation of matter. Proceedings of Royal Society (London) A, 273(1352), 1-11.

[17] Hoyle, F. and Narlikar, J.V. (1964) The $C$-field as a direct particle field. Proceedings of Royal Society (London) A, 282(1389), 178-183.

[18] Chatterjee, S. and Banerjee, A. (2004) C-field cosmology in higher dimensions. General Relativity Gravitation 36(2), 303-313.

[19] Singh, T. and Chaubey, R. (2009) Bianchi type-I, III, V, VI and Kantowski-Sachs universes in creation-field cosmology. Astrophysical Space Science, 321, 5. 